\title{
Approaches to the demonstration of congenital heart disease
}

\author{
GA RUSSELL， *PJ BERRY \\ From the Department of Histopathology, Addenbrooke's Hospital, Cambridge and the *Paediatric Pathology \\ Department, Bristol Royal Hospital for Sick Children, Bristol
}

SUMMARY Advances in antenatal screening techniques have increased the interest in obtaining a detailed pathological correlation with the ultrasonographic findings obtained before death. As a consequence, inadequacies in traditional methods used by pathologists to display congenital malformations have been brought to light. We describe a simple technique of inflation and wax impregnation for the permanent proof of congenital heart defects that can be used in routine perinatal necropsies.

Special approaches to the fetal and perinatal necropsy, which differ from those used for adult work, have been well documented. ${ }^{12}$ The necessity for such methods is a reflection of the range of complex malformations, subtle disease, and iatrogenic processes unique to this age group. Methods for the examination of the heart in these cases have received relatively little attention. The lesions of congenital heart disease are often complex and difficult to display effectively without destroying the anatomical relations that aid their recognition. Clinicians tend to visualise the heart in terms of angiographic images or "slices" from the 2-D echocardiogram. The time honoured methods used by pathologists to open the heart are of little value in these difficult cases, as the distortion of the organ and its anomalies make subsequent clinical correlation formidable. It would seem more appropriate to process and dissect a heart in a way that permits an effective proof of its anomalies and correlation with investigations before death.

The process of inflation and wax impregnation is not new; it was first described by Gross and Leslie in $1931 .{ }^{3}$ Although the method has been widely used in the United States of America, it is not as well known in this country. We reapplied the technique to the analysis of congenital heart disease and found it to be a valuable adjunct to paediatric necropsy.

\section{Methods}

INFLATION OF THE HEART

The heart is first inspected in situ. Left superior vena

Accepted for publication 18 December 1985 cava and anomalies of the pulmonary venous drainage are specifically sought as these lesions may be destroyed by subsequent dissection. The major arterial and venous connections of the heart are then identified and ligated with suture. In a small heart this can be a delicate and time consuming procedure. Alternative methods, however, such as using arterial clamps give much less effective results. The vessels are ligated as far away from the heart as possible to retain the maximum length of vessel still attached to the organ. The inferior vena cava is tied off below the diaphragm, and the pulmonary veins are tied off out-

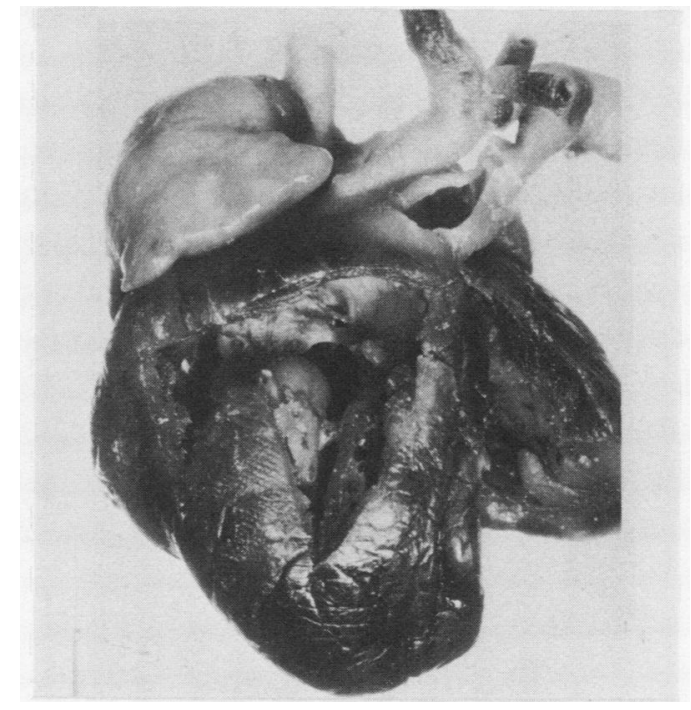

Fig. 1 Congenital rhabdomyoma on papillary muscle of right ventricle from child with tuberous sclerosis. Note artefactual distension of right atrium. 


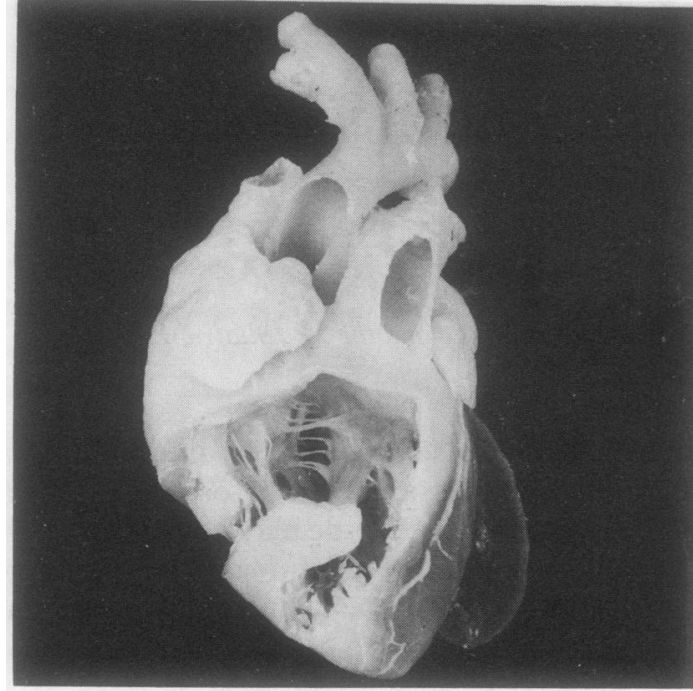

Fig. 2 Wax impregnated heart from 4 month old baby showing windows cut out to show normal anatomy of right ventricle and great vessels.

side the pericardial sac. The only vessel left open is the superior vena cava, which is now incised below the brachiocephalic vein. A fine bore cannula is introduced into the superior vena cava. This tube is manoeuvered into the right atrium and then, via the tricuspid valve, into the right ventricle. The vena cava is tied off around the cannula, and the same length of the suture is tied around the syringe end of the can-

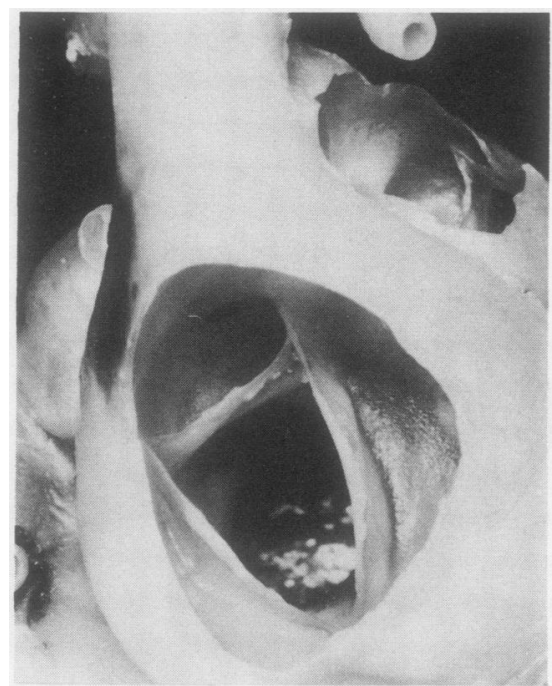

Fig. 3 Aortic root dilatation from child with Marfan's syndrome. Traditional techniques for opening heart would have disrupted valve ring.

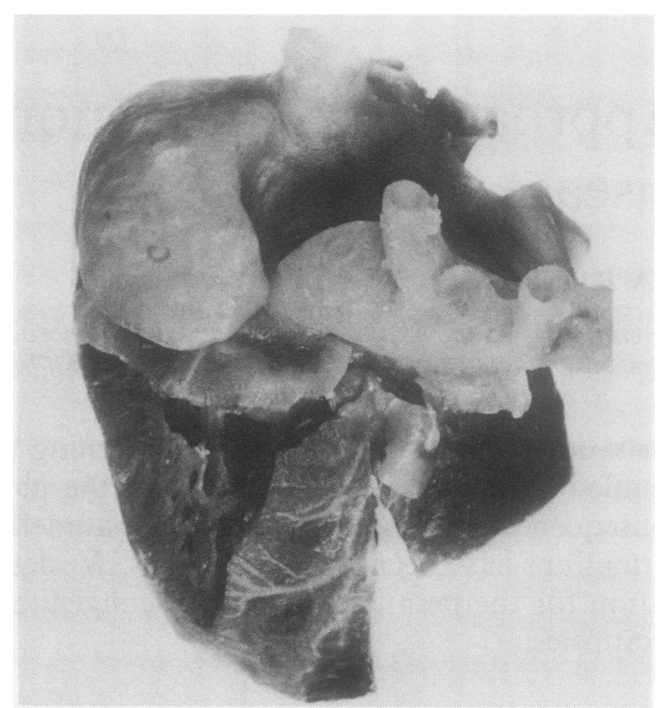

Fig. 4 Infundibular and pulmonary valve stenosis in case of Fallot's tetralogy.

nula. This allows the suture to bear the weight of the specimen, rather than the cannula, when it is being suspended and inflated. Otherwise the cannula tends to slide out of the specimen. The system is then tested for leaks by injecting with physiological saline. If the heart is placed on coloured filter paper small leaks may be rapidly identified by the resulting discolouration of the wetted paper. Leaks are tied off by

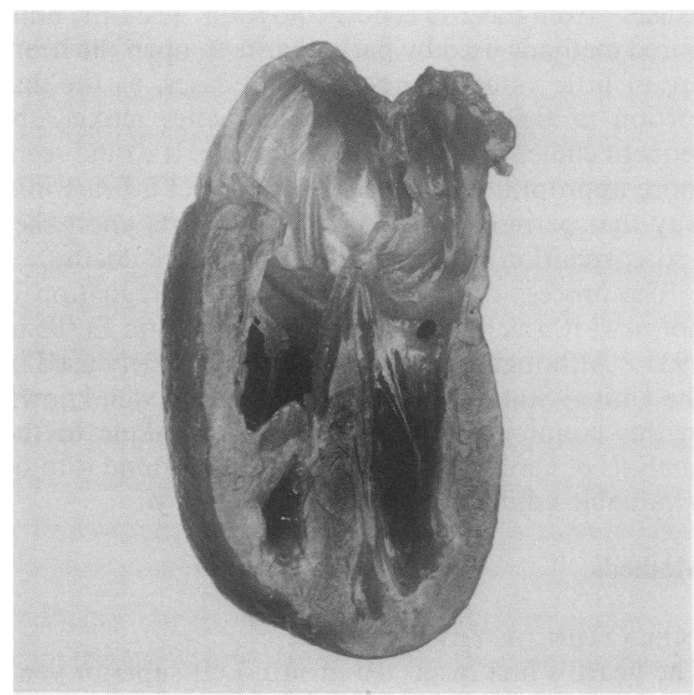

Fig. 5 Heart sectioned along plane equivalent to four chamber view used by echocardiographers. Heart is from 32 week fetus. Notice blood cyst on mitral valve. 

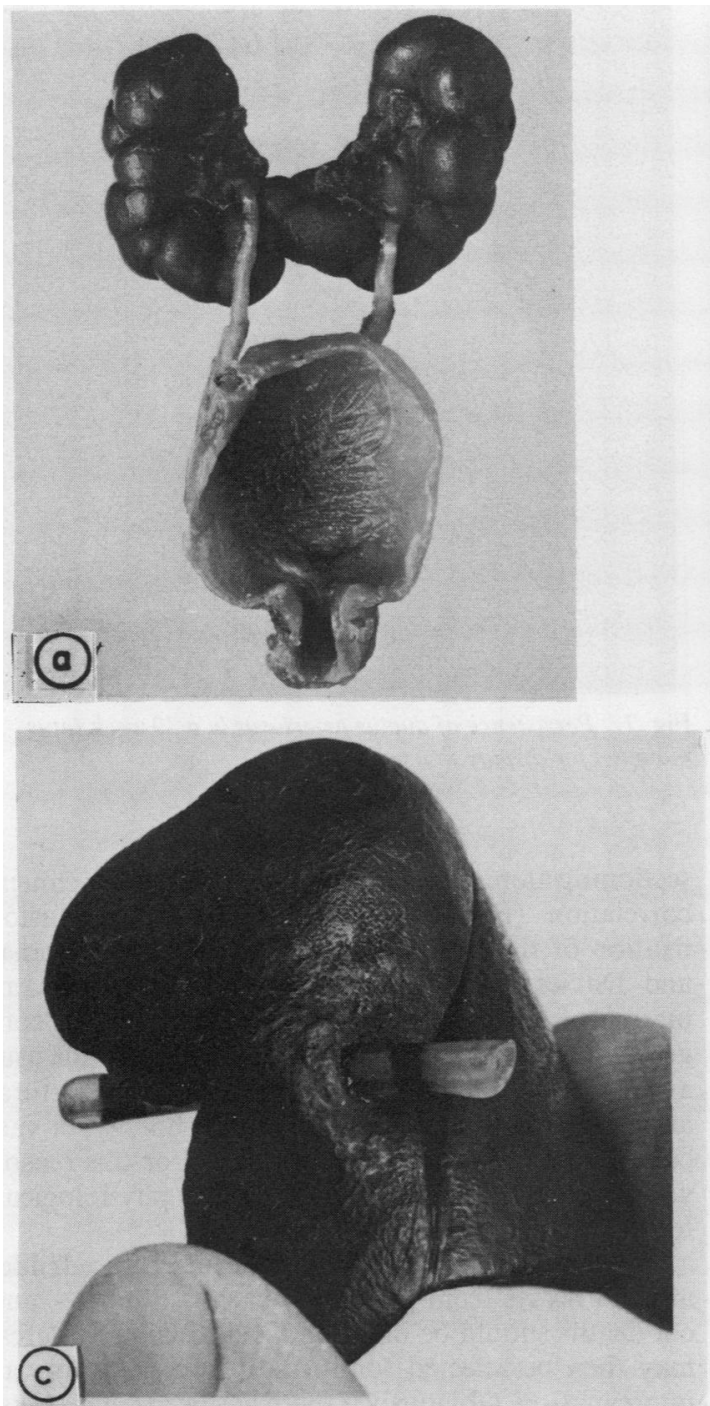
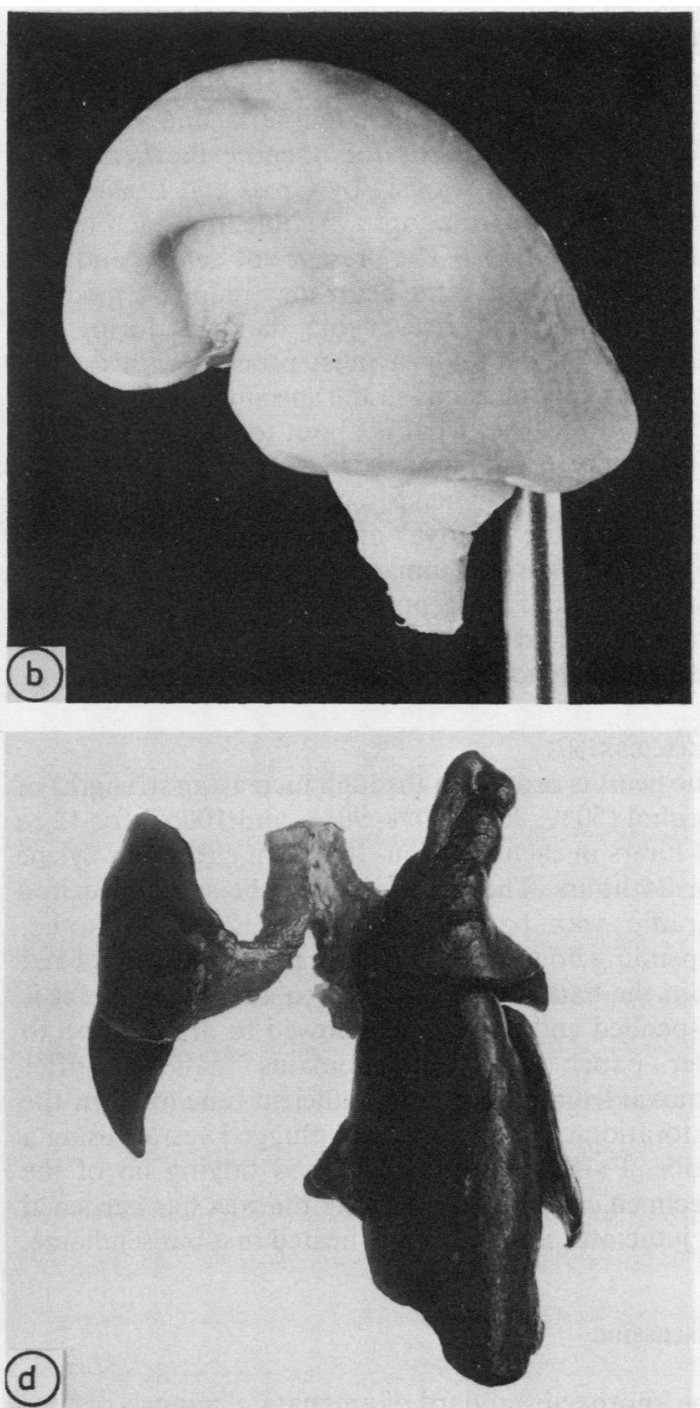

Fig. 6 Wax impregnation methods used on other fetal organs: (a) horsehoe kidney and distended bladder due to posterior urethral valves (32 week fetus with Edward's syndrome); (b) brain from 19 week fetus; (c) lung from 28 week premature baby with hyaline membrane disease impaled by chest drain; (d) hypoplastic left lung in a case of diaphragmatic hernia. further pieces of suture, or carefully, with fine mosquito clamps.

The cannula is then attached to tubing, and a tank of fixative. Ten per cent formalin provides adequate fixation and retention of the inflated shape if left overnight. A more rapid fixation is obtained by using a mixture of absolute alcohol and $40 \%$ formalin (4/1); care, however, must be taken not to get this on to the skin as it is an extreme irritant. The tank is left about
$50 \mathrm{~cm}$ above the height of the specimen, which floats in a bucket of the same fixative.

The method described leads to some artefactual overdistension of the right atrium and coronary sinus, but this does not detract from the end result (Fig. 1). This simple perfusion method works only in fetal and perinatal hearts because of the communications between right and left sides via the foramen ovale and ductus. 


\section{DISSECTION}

Once fixed, the heart is disconnected from the tubing and the ties removed. Windows are cut in the chambers to show both normal landmarks and any anomalies (Fig. 2). Methods for opening the heart have been described previously by Gross and Leslie. ${ }^{4}$ It is better, however, to adopt a flexible approach to cutting these windows. The presence of defects and anatomical variation in the heart may require a modified method to avoid irrevocable damage during dissection. The pathologist must proceed with caution while making incisions in the specimen, pausing continually to inspect what is about to be cut. The valve rings and valve apparatus are always left intact (Figs. 3 and 4). The specimen can also be "tidied up" at this stage with the removal of any remaining pieces of diaphragm, pericardium, fat, or adventitia. Photography is essential in documenting congenital heart malformations and is best performed at this stage as processing tends to fade the colours of the specimen.

\section{PROCESSING}

The heart is processed through increasing strengths of alcohol $(50 \%, 70 \%, 80 \%, 90 \%$, and $100 \%)$ for 12 to 24 hours in each solution. It is then cleared in xylene for 24 hours. The heart can then be suspended in a paraffin wax bath at $60^{\circ} \mathrm{C}$ for 12 to 24 hours, depending on the size of the organ. It is then removed from the bath and transferred to an oven where it is suspended and excess wax allowed to drain off on to filter paper. The heart remains malleable after removal from the oven for sufficient time to allow the restoration of patency to wax plugged vessels using a probe. Further improvement and tidying up of the specimen can be achieved after the wax has hardened by judicious use of a probe, heated in a bunsen flame.

\section{Discussion}

The improved standard of antenatal diagnosis of congenital malformations by ultrasonography and amniocentesis has increased the number of therapeutic abortions requiring careful examination by pathologists. Not only must the pathologist confirm the original diagnosis but he must also assess the degree and range of anomaly associated with a particular chromosomal abnormality. Pathological examination provides an essential form of audit for this most drastic therapeutic procedure. Congenital heart disease can also be detected antenatally at specialist centres using high resolution ultrasonography. ${ }^{5}$

Inflation fixation of the heart shows anomalies with much greater clarity as the chambers acquire an anatomical capacity and shape. The inflated specimen can be dissected to suit any specific requirement including

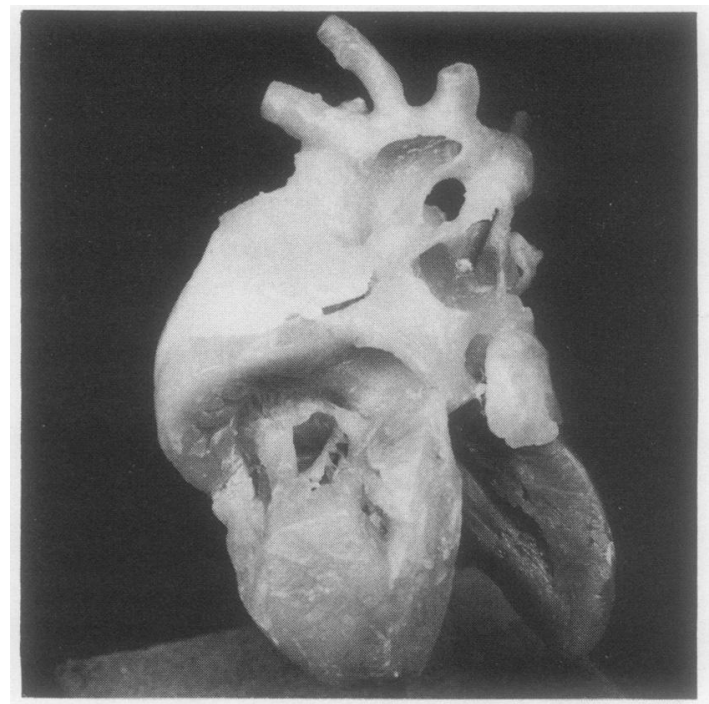

Fig. 7 Persistence of ductus arteriosus in a 39 week fetus mounted for display in a museum.

sectioning along echocardiographic planes for clinical correlation (Fig. 5). ${ }^{6}$ A technique for perfusion fixation of the heart has been described by Thomas and Davies. ${ }^{7}$ Their method permits careful examination of valve cusps in diastolic positions. In congenital heart disease valvular competence is not usually a source of concern. Rather, it is gross structural anomalies of septation, chamber morphology, or vascular connection that are of interest. For this reason we adopted the simpler, though less physiological, technique of Gross and Leslie.

In which cases should this procedure be used? Ideally, all hearts from perinatal deaths, stillbirths, and cot deaths should be inflation fixed. Cases of interest $\delta$ may then be selected for further processing to wax impregnation. Obviously if there has been clinical sus- 0 picion of heart disease in life or if the external morphology of the heart is abnormal then inflation should be performed. Babies born to mothers at risk of developing congenital heart disease-for example, $\Omega$ by virtue of maternal diabetes or high alcohol $\tilde{O}$ intake-should also be examined by this method.

Wax impregnation need not be confined to the 0 heart (Fig. 6). Any organ may be processed in this way, although modifications to the method may be required for more delicate organs such as the fetal $\stackrel{\oplus}{?}$ brain. Abnormalities in the finished specimen may be 0 highlighted by the use of coloured probes or pins. They can be handled without the need for gloves at $\stackrel{\mathbb{Q}}{\Omega}$ clinical meetings or teaching sessions and are also $\vec{D}$ suitable for display in museums. (Fig. 7). Storage is $\frac{a}{\sigma}$ 
simple - small drawer filing boxes of the sort used to keep photographic slides are ideally suited when labelled and lined with cotton wool.

We are indebted to Drs BE Favara and CR Hawes of the Children's Hospital, Denver, who introduced us to this method. We thank Mr C Burton for photographic help.

\section{References}

${ }^{1}$ Pryse-Davies J. The perinatal autopsy. In: Anthony P, MacSween $\mathrm{R}$, eds. Recent advances in histopathology. London: Churchill Livingstone, 1981:65-82.

${ }^{2}$ Barson AJ. The perinatal postmortem. In: Barson AJ, ed. Fetal and neonatal pathology. Eastbourne: Praeger, 1981:149-66.
${ }^{3}$ Gross L, Leslie E. Paraffin infiltration of the heart. Am Heart $J$ 1931;6:665-71.

${ }^{4}$ Titus JL. The heart and vascular system. In: Ludwig J, ed. Current methods of autopsy practice. Philadelphia: WB Saunders, 1979:51-92.

${ }^{5}$ Allan LD, Tynan MJ, Campbell S, Anderson RH. Normal fetal cardiac anatomy - a basis for echocardiographic detection of abnormalities. Prenatal Diagnosis 1981;1:131-9.

${ }^{6}$ Allan LD, Tynan MJ, Campbell S, Wilkinson JL, Anderson RH. Echocardiographic and anatomical correlates in the fetus. $\mathrm{Br}$ Heart $J$ 1980;44:441-51.

7 Thomas AC, Davies MJ. The demonstration of cardiac pathology using perfusion fixation. Histopathology 1985;9:5-19.

Requests for reprints to: Dr GA Russell, Department of Histopathology, Addenbrooke's Hospital, Cambridge CB2 2QQ. 